\title{
NONCROSSED PRODUCT DIVISION ALGEBRAS WITH A BAER ORDERING
}

\author{
PATRICK J. MORANDI AND B. A. SETHURAMAN
}

(Communicated by Ken Goodearl)

\begin{abstract}
Let $n \mid m$ be positive integers with the same prime factors, such that $p^{3} \mid n$ for some prime $p$. We construct a noncrossed product division algebra $D$ with involution $*$, of index $m$ and exponent $n$, such that $D$ possesses a Baer ordering relative to the involution $*$. Using similar techniques we construct indecomposable division algebras with involution possessing a Baer ordering.
\end{abstract}

\section{INTRODUCTION}

If $D$ is either a field or a division ring equipped with an involution * (an anti-automorphism of order 2), a Baer ordering on $D$ is a subset $P$ of the set of symmetric elements of $D$ under $*$ such that (1) $P+P \subseteq P$, (2) $P$ contains 1 but not 0 , (3) $d P d^{*} \subseteq P$ for every $d \in D-\{0\}$, and (4) if $d \neq 0$ is a symmetric element of $D$, then either $d \in P$ or $-d \in P$. These orderings were first defined by Baer $[B]$, and later studied by Holland and others (cf. $\left[\mathrm{H}_{1}\right],\left[\mathrm{H}_{2}\right],\left[\mathrm{H}_{3}\right],\left[\mathrm{C}_{1}\right]$, $\left.\left[\mathrm{C}_{2}\right],\left[\mathrm{C}_{3}\right],\left[\mathrm{Le}_{1}\right],\left[\mathrm{Le}_{2}\right],[\mathrm{MW}]\right)$. The following question arose during discussions with Holland: do there exist noncrossed product division algebras which possess Baer orderings? The purpose of this paper is to answer this question in the affirmative. We show that for every pair of positive integers $n \mid m$ having the same prime factors, such that $p^{3} \mid n$ for some prime $p$, then there is a noncrossed product division algebra of index $m$ and exponent $n$ possessing a Baer ordering. Furthermore, for any prime $p$, if $m \geq 3$ and $n$ satisfies $m \geq n \geq \frac{m}{2}+1$, we construct an indecomposable division algebra of index $p^{m}$ and exponent $p^{n}$ possessing a Baer ordering. The method we use to produce noncrossed products and indecomposable division algebras is that developed in [JW]; that is, our examples will be the underlying division algebra of a tensor product of suitably chosen symbol algebras over a field $F=L_{1} \cap \cdots \cap L_{k}$, where the $L_{i}$ are strictly $p$-Henselian fields. The involutions on our examples are necessarily of the second kind, since a division ring $D$ has an involution of the first kind iff the exponent of $D$ is 2 . As for division algebras with an involution of the first kind, an indecomposable division algebra of exponent 2

Received by the editors July 20, 1993 and, in revised form, November 17, 1993.

1991 Mathematics Subject Classification. Primary 16K20, 12J20, 16W10.

Both authors were supported in part by the National Science Foundation. 
is constructed in [CW], and that example contains a Baer ordering, as pointed out in the remark below after Proposition 11. It is unknown whether there exist noncrossed products of exponent 2.

\section{2. *-VALUATIONS AND BAER ORDERED DIVISION RINGS}

We recall the "lifting criterion" from $\left[\mathrm{H}_{2}\right]$, which we will use to construct Baer orderings on our examples below. If $D$ is a division ring with an involution $*$, then a valuation $v$ on $D$ is said to be a $*$-valuation if $v\left(d^{*}\right)=v(d)$ for all $d \in D^{\times}=D-\{0\}$. That is, $v$ is a $*$-valuation if $v \circ *=v$. If $\bar{D}$ is the residue division ring of $D$ relative to a *-valuation, then $\bar{D}$ has an involution induced from $*$, which we will also denote by $*$, defined by $\bar{u}^{*}=\overline{u^{*}}$.

Let $d$ be either a symmetric or a skew-symmetric element of $D^{\times}$. Then $d^{*} d^{-1} \in Z(D)$, the center of $D$, and it is easy to check that the map $x \mapsto$ $d x^{*} d^{-1}$ is also an involution of $D$, and induces an involution on $\bar{D}$. Let $c_{d}$ be the automorphism on $\bar{D}$ induced by conjugation by $d$. The element $d$ is said to be smooth if the residue involution $c_{d} \circ *$ is equal to $\varphi \circ * \circ \varphi^{-1}$ for some automorphism $\varphi$ of $\bar{D}$.

Now suppose that $v$ is a $*$-valuation on $D$ with $v(2)=0$, that is, $\operatorname{char}(\bar{D}) \neq$ 2 . It can be seen that for every $\gamma \in \Gamma_{D}$, the value group of $D, v^{-1}(\gamma)$ either contains a symmetric or skew-symmetric element (see $\left.\left[\mathrm{H}_{2}, \S 3\right]\right)$. A *-valuation $v$ on $D$ is said to be smooth if (1) $v(2)=0$, and (2) for every $\gamma \in \Gamma_{D}$, if $v^{-1}(\gamma)$ contains a symmetric element, then it contains a smooth symmetric element, else $v^{-1}(\gamma)$ contains a smooth skew-symmetric element.

The following was proved by Holland.

Theorem 1 [ $\mathrm{H}_{2}$, Corollary 3.3]. If $\mathrm{D}$ is a division ring with involution $*$, and if $v$ is a smooth *-valuation on $D$, then every Baer ordering on $\bar{D}$ defined with respect to the residue involution * on $\bar{D}$ lifts to a Baer ordering on $D$ with respect to $*$.

We will apply the theorem above to obtain division algebras with a Baer ordering. The following corollary summarizes our technique.

Corollary 2. Let $F / K$ be a separable quadratic field extension and $D$ an $F-$ central division algebra such that the corestriction $\operatorname{Cor}_{F / K}(D)=0$. Suppose $D$ has a valuation $v$ such that $D / F$ is totally ramified and $F / K$ is inertial. Also suppose that the residue field $\bar{K}$ has an ordering which does not extend to $\bar{F}$. Then there is an involution * on $D$ which is trivial on $K$ such that $v$ is a *-valuation, and the ordering on $\bar{K}$ lifts to a Baer ordering on $D$ with respect to $*$.

Proof. By Scharlau's theorem [D, p. 118], $D$ has an involution * which extends the nonidentity automorphism $\sigma$ of $\operatorname{Gal}(F / K)$. The valuation $\left.v\right|_{K}$ extends uniquely to $F$ by hypothesis, so $v$ is a $*$-valuation since $\left.v \circ *\right|_{K}=\left.v\right|_{K}$. Note that $v(2)=0$ since $\operatorname{char} \bar{K}=0$. Furthermore, $v$ is smooth, because $c_{d}=\mathrm{id}$ for any $d \in D^{\times}$as $\bar{D}=\bar{F}$. To apply Theorem 1 , it suffices to show that the ordering on $\bar{K}$ is a Baer ordering on $\bar{D}$ with respect to the residue involution $\bar{*}$. Since $F / K$ is quadratic and inertial, $\bar{F}=\bar{K}(\sqrt{a})$ for some $a \in \bar{K}$. If $P$ is the ordering on $\bar{K}$, then $a \notin P$ since $P$ does not extend to $\bar{F}$. If $x=\alpha+\beta \sqrt{a} \in \bar{F}$, then $x x^{\bar{*}}=\alpha^{2}-\beta^{2} a>0$, hence property (3) of the 
definition of a Baer ordering is satisfied for $P$. The other properties of a Baer ordering are trivial to check, so $P$ is indeed a Baer ordering of $\bar{D}$ with respect to $\bar{*}$.

\section{INTERSECTICNS OF STRICTLY $p$-HENSELIAN VALUED FIELDS}

Let $p$ be a prime, and let $F$ be a field containing a primitive $p$ th root of unity $\xi$. Let $F(p)$ be the compositum inside an algebraic closure of $F$ of all Galois extensions $K$ of $F$ with $[K: F]$ a power of $p .(F(p)$ is called the $p$-closure of $F$.) Let $v$ be a valuation on $F$. Recall that $F$ is said to be $p$-Henselian if $\operatorname{char}(\bar{F}) \neq p$ and $v$ has a unique extension to $F(p)$.

A valued field $(F, v)$ with $\xi \in F$ is said to be strictly $p$-Henselian if it is $p$-Henselian and $\bar{F}=\bar{F}(p)$. Note that if $(F, v)$ is strictly $p$-Henselian, then the valuation units of $F$ are $p^{r}$ th powers in $F$ for any $r$, and $\operatorname{Gal}(F(p) / F) \cong$ $\oplus_{i=1}^{n} \widehat{\mathbb{Z}}_{p}$, where $n=\operatorname{dim}_{\mathbb{Z} / p \mathbb{Z}}\left(\Gamma_{F} / p \Gamma_{F}\right)$, and $\widehat{\mathbb{Z}}_{p}$ is the $p$-adic integers (see [JW, Lemma 1.9]).

If $(F, v)$ is a valued field with $\operatorname{char}(\bar{F}) \neq p$, fix an extension of $v$ to $F(p)$, and let $L$ be the fixed field of the inertia group of $F(p)$. Then $\left(L,\left.v\right|_{L}\right)$ is called the strict $p$-Henselization of $(F, v)$. The valued field $L$ is strictly $p-$ Henselian, and is a maximal unramified extension of $F$ in $F(p)$. Moreover, the strict $p$-Henselization of a field is unique up to isomorphism.

Recall that if $F$ is any field with valuations $v_{1}, \ldots, v_{k}$, then these valuations are said to be pairwise independent if no proper subring of $F$ contains the valuation rings of any pair of valuations $v_{i}, v_{j}$. If $F$ is a field containing a primitive $p$ th root of unity and $A$ is a central simple $F$-algebra of exponent a power of $p$, the $p$-index of $A$ is defined to be

$$
p-\operatorname{ind}(A)=\min \{[L: F] \mid F \subseteq L \subseteq F(p), L \text { splits } A\} .
$$

Note that $\operatorname{ind}(A) \leq p-\operatorname{ind}(A)$, with equality whenever $A$ has a maximal subfield contained in $F(p)$. The following was proved by Jacob and Wadsworth [JW, Theorem 4.11].

Theorem 3 (Jacob-Wadsworth). Let $F_{0}$ be a field with pairwise independent valuations $v_{1}, \ldots, v_{k}$, containing a primitive pth root of unity. Let $L_{j}$ be a strict $p$-Henselization of $\left(F_{0}, v_{j}\right)$, and let $F=L_{1} \cap \cdots \cap L_{k}$. With respect to $v_{j}$ we have $\bar{F}=\overline{L_{j}}$ and $\Gamma_{F}=\Gamma_{L_{j}}$, and for any central simple $F$-algebra $A$ of exponent a power of $p$, we have

(1) $\operatorname{ind}(A) \leq p-\operatorname{ind}(A)=\max \left\{p-\operatorname{ind}\left(A \otimes_{F} L_{j}\right) \mid 1 \leq j \leq k\right\}$;

(2) if $\operatorname{ind}\left(A \otimes_{F} L_{j}\right)=p-\operatorname{ind}\left(A \otimes_{F} L_{j}\right)$ for each $j$, then $\operatorname{ind}(A)=$ $p$-ind $(A)$.

Proof. This is really just an amalgamation of Theorems 4.3, Remark 4.4(ii) and Theorem 4.11, as well as the remark at the end of $\S 4$ of [JW].

\section{THe EXAMPLes}

We start this section by constructing noncrossed products containing a Baer ordering, of index $p^{m}$ and exponent $p^{n}$. Let $p$ be a prime and $m \geq n \geq 3$ integers. Let $F_{0}=\mathbb{C}\left(x_{1}, x_{2}, y_{1}, \ldots, y_{2 m}\right)$, where the $x_{j}$ and $y_{j}$ are indeterminates. Recall that if $L$ is any field and $z_{1}, \ldots, z_{k}$ are indeterminates, 
then ordering $\Gamma=\oplus_{j=1}^{k} \mathbb{Z}$ anti-lexicographically, there is a unique valuation $v: L\left(z_{1}, \ldots, z_{k}\right)^{\times} \rightarrow \Gamma$ such that $v\left(z_{j}\right)=(0, \ldots, 0,1,0, \ldots, 0)$ (the 1 is in the $j$ th place) and $v(l)=0$ for all $l \in L^{\times}$. We refer to this valuation as the $\left(z_{1}, \ldots, z_{k}\right)$-adic valuation on $L\left(z_{1}, \ldots, z_{k}\right)$. Let $v_{1}$ be the $\left(x_{1}, x_{2}\right)$-adic valuation on $F_{0}$ (viewed as a rational function field over $\left.\mathbb{C}\left(y_{1}, \ldots, y_{2 m}\right)\right)$ and $v_{2}$ the $\left(y_{1}, \ldots, y_{2 m}\right)$-adic valuation on $F_{0}$ (viewed as a rational function field over $\left.\mathbb{C}\left(x_{1}, x_{2}\right)\right)$. Then the value group and residue field of $F_{0}$ with respect to $v_{1}$ are $\mathbb{Z} \oplus \mathbb{Z}$ and $\mathbb{C}\left(y_{1}, \ldots, y_{2 m}\right)$ respectively. Similarly, the value group and residue field of $F_{0}$ with respect to $v_{2}$ are $\oplus_{j=1}^{2 m} \mathbb{Z}$ and $\mathbb{C}\left(x_{1}, x_{2}\right)$ respectively.

Let $\sigma$ be the automorphism of $F_{0}$ extending complex conjugation on $\mathbb{C}$ defined by $\sigma\left(x_{1}\right)=x_{2}, \sigma\left(x_{2}\right)=x_{1}$ and $\sigma\left(y_{j}\right)=y_{j}$. Then $\sigma$ is an automorphism of order 2 , and the fixed field is easily seen to be

$$
K_{0}:=\mathbb{R}\left(x_{1}+x_{2}, i\left(x_{1}-x_{2}\right), y_{1}, \ldots, y_{2 m}\right) .
$$

Therefore $F_{0}=K_{0}(i)$. Note that with respect to the restriction of $v_{2}$ to $K_{0}$, the residue field $\overline{K_{0}}$ is $\mathbb{R}\left(x_{1}+x_{2}, i\left(x_{1}-x_{2}\right)\right)$, a purely transcendental extension of $\mathbb{R}$. This implies that $\overline{K_{0}}$ is formally real, hence has an ordering $\mathscr{P}$. Then by [L, Theorem 3.10], there is an ordering $P$ on $K_{0}$ which is compatible with $v_{2}$ in the sense of $[\mathrm{L}, \S 2]$. Let $C$ be a fixed algebraic closure of $F_{0}$, and extend $v_{1}$ and $v_{2}$ to valuations on $C$, which we also denote by $v_{1}$ and $v_{2}$. Let $K^{\prime}$ be a Henselization of $K_{0}$ inside $C$ with respect to $v_{2}$. There is an ordering $Q$ on $K^{\prime}$ extending $P$ which is compatible with $v_{2}$ by [L, Theorem 3.16, Corollary 3.22]. Let $R$ be a real closure of $\left(K^{\prime}, Q\right)$ inside $C$. Since $C=R(i)$ and $F_{0}=K_{0}(i)$, the automorphism of $C$ that fixes $R$ and sends $i$ to $-i$ is an extension of $\sigma$ on $F_{0}$, and we will denote it also by $\sigma$. With respect to $\left.v_{2}\right|_{R}$, the field $R$ is Henselian, since $R$ is an algebraic extension of the Henselian field $K^{\prime}$. Thus the ordering $R^{2}$ of $R$ is compatible with $\left.v_{2}\right|_{R}$. Furthermore, since $\left(R, v_{2}\right)$ is Henselian, the valuation $v_{2}$ on $R$ extends uniquely to $C$, hence $v_{2} \circ \sigma=v_{2}$.

Let $F(p)$ be the $p$-closure of $F_{0}$ inside $C$, and let $L_{1}$ and $L_{2}$ be strict $p$-Henselizations inside $F(p)$ of $\left(F_{0}, v_{1}\right)$ and $\left(F_{0}, v_{2}\right)$ respectively. The field $\sigma\left(L_{j}\right)(j=1,2)$ is then a strict $p$-Henselization of $F_{0}$ with respect to $v_{j} \circ \sigma$ by [E, 19.10]. But since $v_{2} \circ \sigma=v_{2}$ on $C$, we see $\sigma\left(L_{2}\right)=L_{2}$. Let $F=$ $L_{1} \cap \sigma\left(L_{1}\right) \cap L_{2}$. Then $\sigma$ restricts to an automorphism of order 2 on $F$; and if $K=F^{\sigma}$ is the fixed field of $\left.\sigma\right|_{F}$, then $K=F \cap R$, so $K$ is an ordered field with ordering $R^{2} \cap K$ compatible with $v_{2}$. Furthermore, as $i \in F_{0}$, we have $i \in F$, so $F=K(i)$. Taking residues with respect to $v_{2}$, we find $\bar{K}$ is an ordered subfield of $\bar{F}$ with $\bar{F}=\bar{K}(i)$. Thus, the ordering on $\bar{K}$ is a Baer ordering on $\bar{F}$ with respect to the residue involution $\bar{\sigma}$ (see the proof of Corollary 2).

Our examples of noncrossed product algebras with Baer ordering will be constructed over the field $F$. The following lemma will allow us to use the localglobal principles of [JW] (Theorem 3 above) to analyze division algebras with center $F$.

Lemma 4. The valuations $v_{1}, v_{1} \circ \sigma$ and $v_{2}$ are independent on $F_{0}$, hence on $F$. 
Proof. Let $V_{j}$ be the valuation ring of $v_{j}$ on $F_{0}(j=1,2)$. Then $\sigma\left(V_{1}\right)$ is the valuation ring of $v_{1} \circ \sigma$. To say that two valuations are independent is to say that the two corresponding valuation rings do not lie in any proper common overring of $F_{0}$. Since $v_{1}$ is the $\left(x_{1}, x_{2}\right)$-adic valuation ring of $F_{0}$, the valuation $v_{1} \circ \sigma$ is easily seen to be the $\left(x_{2}, x_{1}\right)$-adic valuation of $F_{0}$. The only proper overring of $V_{1}$ in $F_{0}$ is the $x_{2}$-adic valuation ring of $F_{0}$. Similarly, the only proper overring of $\sigma\left(V_{1}\right)$ in $F_{0}$ is the $x_{1}$-adic valuation ring. Since the $x_{1}$-adic and $x_{2}$-adic valuation rings of $F_{0}$ are distinct, $v_{1}$ and $v_{1} \circ \sigma$ are independent. Clearly $v_{1}$ and $v_{2}$ are independent, as are $v_{1} \circ \sigma$ and $v_{2}$. The field $F$ is an algebraic extension of $F_{0}$, so it follows that the valuations $v_{1}$, $v_{1} \circ \sigma$ and $v_{2}$ are pairwise independent on $F$.

If $L$ is a field containing a primitive $t$ th root of unity $\xi$ and $a, b \in L^{\times}$, let $A_{\xi}(a, b ; L)$ be the (central simple) $L$-algebra of dimension $t^{2}$ generated by two elements $r$ and $s$ subject to the relations

$$
r^{t}=a, \quad s^{t}=b, \quad s r=\xi r s .
$$

The algebra $A_{\xi}(a, b ; L)$ is called a symbol algebra. Our examples will be constructed using symbol algebras. Let $m \geq n \geq 3$ be integers, let $\omega$ be a primitive $p^{n}$ th root of unity in $F$ and $\rho=\omega^{p^{n-1}}$, a primitive $p$ th root of unity. Define

$$
A_{1}=A_{\omega}\left(x_{1}+x_{2}, x_{1} x_{2} ; F\right), \quad A_{2}=\bigotimes_{j=1}^{m} A_{\rho}\left(y_{2 j-1}, y_{2 j} ; F\right) .
$$

Finally, let $D$ be the underlying division algebra of $A_{1} \otimes_{F} A_{2}$. The following lemma is the analogue of [JW, Lemma 5.3] for our situation.

Lemma 5. With notation above, $A_{1} \otimes_{F} L_{1}$ and $A_{1} \otimes_{F} \sigma\left(L_{1}\right)$ are division algebras of index, $p$-index and exponent $p^{n}$, while $L_{1}$ and $\sigma\left(L_{1}\right)$ split $A_{2}$. Also, $A_{2} \otimes_{F} L_{2}$ is a division algebra of index and $p$-index $p^{m}$ and exponent $p$, while $L_{2}$ splits $A_{1}$. Moreover, if $M$ is a splitting field of $A_{2}$ containing $L_{2}$, then $\operatorname{Gal}\left(M / L_{2}\right)$ has $(\mathbb{Z} / p \mathbb{Z})^{m}$ as a homomorphic image.

Proof. Since the $y_{j}$ are units with respect to $v_{1}$, they become $p^{n}$ th powers in $L_{1}$ ([JW, Lemma 1.9]), so $L_{1}$ splits $A_{2}$. We have

$$
A_{1} \otimes_{F} L_{1}=A_{\omega}\left(x_{1}\left(1+x_{1}^{-1} x_{2}\right), x_{1} x_{2} ; L_{1}\right)=A_{\omega}\left(x_{1}, x_{2}\right),
$$

since $1+x_{1}^{-1} x_{2}$ is a unit and $A_{\omega}\left(x_{1}, x_{1}\right)=A_{\omega}\left(x_{1},-x_{1}\right)$ is split. Thus, by [JW, Corollary 2.6], $A_{1} \otimes_{F} L_{1}$ is a totally ramified division algebra with respect to $v_{1}$ of index, $p$-index, and exponent $p^{n}$. Similar arguments apply over the field $\sigma\left(L_{1}\right)$ and $L_{2}$. The proof of the last statement of the lemma is identical to that of the corresponding statement of [JW, Lemma 5.3].

Theorem 6. The division algebra $D$ is a noncrossed product division algebra of index $p^{n}$ and exponent $p^{m}$.

Proof. The index of $D$ follows from Theorem 3 and Lemma 5. As for the exponent, we have $p^{m}=\exp \left(A_{1} \otimes_{F} L_{1}\right)=\exp \left(D \otimes_{F} L_{1}\right) \leq \exp (D)=p^{m}$. The proof that $D$ is a noncrossed product is identical to that of [JW, Theorem 5.4(ii)]. 
We next show that $D$ has an involution $*$, and that the valuation $v_{2}$ extends to a *-valuation on $D$. We do this in the next two lemmas.

Lemma 7. Let $a, b \in K^{\times}$, and let $\xi$ be a primitive th root of unity in $F$. Then the symbol algebra $A_{\xi}(a, b ; F)$ has an involution extending $\sigma$. Therefore there is an involution * on $D$ extending $\sigma$.

Proof. Let $r$ and $s$ be the generators of $A=A_{\xi}(a, b ; F)$ satisfying $r^{t}=a$, $s^{t}=b$, and $s r=\xi r s$. We define $*$ on $A$ by $\left(\sum c_{\alpha \beta} r^{\alpha} s^{\beta}\right)^{*}=\sum \sigma\left(c_{\alpha \beta}\right) \xi^{\alpha \beta} r^{\alpha} s^{\beta}$, where $c_{\alpha \beta} \in F$. Clearly $*$ is additive and extends $\sigma$. Moreover, if $c \in F$, since $\sigma(\xi)=\xi^{-1}$, we see that

$$
\left(c r^{\alpha} s^{\beta}\right)^{* *}=\left(\sigma(c) \xi^{\alpha \beta} r^{\alpha} s^{\beta}\right)^{*}=c \xi^{-\alpha \beta} \xi^{\alpha \beta} r^{\alpha} s^{\beta}=c r^{\alpha} s^{\beta} .
$$

Therefore $*$ has order 2 . To see that $*$ is an anti-automorphism, it suffices to show that $\left(r^{\alpha} s^{\beta} \cdot r^{\gamma} s^{\delta}\right)^{*}=\left(r^{\gamma} s^{\delta}\right)^{*}\left(r^{\alpha} s^{\beta}\right)^{*}$. Now $r^{\alpha} s^{\beta} \cdot r^{\gamma} s^{\delta}=\left(\xi^{\beta \gamma} r^{\alpha+\gamma} s^{\beta+\delta}\right)^{*}$, so

$$
\left(r^{\alpha} s^{\beta} \cdot r^{\gamma} s^{\delta}\right)^{*}=\xi^{-\beta \gamma+(\alpha+\gamma)(\beta+\delta)} r^{\alpha+\gamma} s^{\beta+\delta},
$$

whereas

$$
\left(r^{\gamma} s^{\delta}\right)^{*}\left(r^{\alpha} s^{\beta}\right)^{*}=\xi^{\gamma \delta} r^{\gamma} s^{\delta} \xi^{\alpha \beta} r^{\alpha} s^{\beta}=\xi^{\gamma \delta+\alpha \beta+\delta \alpha} r^{\gamma+\alpha} s^{\delta+\beta}
$$

Thus these two elements are equal, so $*$ is in fact an involution of $A$ extending $\sigma$.

To see that $D$ has an involution * extending $\sigma$ on $F$, by the argument above, each of $A_{\omega}\left(x_{1}+x_{2}, x_{1} x_{2} ; F\right), A_{\rho}\left(y_{2 j-1}, y_{2 j} ; F\right)(1 \leq j \leq m)$ has an involution extending $\sigma$. Therefore by Scharlau's theorem [D, p. 118], if cor is the corestriction map from $F$ to $K$, we see each of these algebras has corestriction zero in $\operatorname{Br}(K)$, hence $\operatorname{cor}(D)=0$. Thus applying Scharlau's theorem again, $D$ has an involution $*$ extending $\sigma$.

Lemma 8. There is a valuation on $D$ extending $v_{2}$ which is a *-valuation. Furthermore, with respect to this valuation, $D / F$ is totally ramified.

Proof. Since $D$ and $A_{2} \otimes_{F} L_{2}$ both have index $p^{m}$, the similarity $D \otimes_{F} L_{2} \sim$ $A_{2} \otimes_{F} L_{2}$ shows that $D \otimes_{F} L_{2}=A_{2} \otimes_{F} L_{2}$. The valuation $v_{2}$ extends to a totally ramified valuation on $A_{2} \otimes_{F} L_{2}$ by [JW, Corollary 2.6], which then restricts to a valuation on $D$, which we will also call $v_{2}$. Since $\bar{F} \subseteq \bar{D} \subseteq$ $\overline{A_{2} \otimes_{F} L_{2}}=\overline{L_{2}}=\bar{F}$, the last equality by Theorem 3 , we see that $\bar{D}=\bar{F}$. Since $\operatorname{char}(\bar{F})=0, D / F$ is defectless with respect to $v_{2}$, so it follows that $D / F$ is totally ramified with respect to $v_{2}$. Finally, $v_{2}$ and $v_{2} \circ *$ are both valuations on $D$ that extend $\left.v_{2}\right|_{F}=\left.v_{2}\right|_{F} \circ \sigma$, hence $v_{2}=v_{2} \circ *$ by [W, Theorem]. Thus $v_{2}$ is a $*$-valuation on $D$.

We are now able to show that the noncrossed product division ring $D$ possesses a Baer ordering relative to the involution $*$.

Corollary 9. If $D$ is the noncrossed product of index $p^{m}$ and exponent $p^{n}$ defined above, then $D$ has a Baer ordering relative to the involution * on $D$ defined in Lemma 7.

Proof. This follows from Corollary 2 , since $D / F$ is totally ramified with respect to the $*$-valuation $v_{2}$, by Lemma 8 , and $\bar{F} / \bar{K}$ is separable quadratic by construction. 
We now construct examples of Baer ordered noncrossed products of arbitrary index $m$ and exponent $n$, provided $p^{3} \mid n$ for some prime $p$. Let $n \mid m$ be positive integers with the same prime factors, say $m=p_{1}^{\beta_{1}} \cdots p_{t}^{\beta_{t}}$ and $n=$ $p_{1}^{\alpha_{1}} \cdots p_{t}^{\alpha_{t}}$, and suppose $\alpha_{1} \geq 3$. Let $I$ be the noncrossed product of index $p_{1}^{\beta_{1}}$ and exponent $p_{1}^{\alpha_{1}}$ with involution $*_{I}$ containing a Baer ordering as constructed in Corollary 9. Let $F=Z(I)$, and let $K$ be the symmetric subfield of $F$. Let $\left\{z_{j}^{(k)} \mid 2 \leq k \leq t, 1 \leq j \leq 2 s_{k}\right\}$ be a set of indeterminates over $F$, where $s_{k}=\beta_{k}-\alpha_{k}+1$, and let $F^{\prime}$ and $K^{\prime}$ be the iterated Laurent series fields $F^{\prime}=F\left(\left(z_{1}^{(1)}\right)\right) \cdots\left(\left(z_{2 s_{t}}^{(t)}\right)\right)$ and $K^{\prime}=K\left(\left(z_{1}^{(1)}\right)\right) \cdots\left(\left(z_{2 s_{t}}^{(t)}\right)\right)$ respectively. Note that $\left[F^{\prime}: K^{\prime}\right]=2$, and the nonidentity automorphism $\sigma$ of $F^{\prime}$ which fixes $K^{\prime}$ extends $\left.*\right|_{F}$. Let $\omega_{k}$ be a primitive $p_{k}^{\alpha_{k}}$-root of unity in $F$ and $\rho_{k}$ a primitive $p_{k}$ th root of unity in $F$. Set

$T_{k}=A_{\omega_{k}}\left(z_{1}^{(k)}, z_{2}^{(k)} ; F^{\prime}\right) \otimes_{F^{\prime}} A_{\rho_{k}}\left(z_{3}^{(k)}, z_{4}^{(k)} ; F^{\prime}\right) \otimes_{F^{\prime}} \cdots \otimes_{F^{\prime}} A_{\rho_{k}}\left(z_{2 s_{k}-1}^{(k)}, z_{2 s_{k}}^{(k)} F^{\prime}\right)$,

and let $T=T_{2} \otimes_{F^{\prime}} \cdots \otimes_{F^{\prime}} T_{t}$. Then with respect to the $\left(z_{1}^{(1)}, \ldots, z_{2 s_{t}}^{(t)}\right)$-adic valuation on $F^{\prime}$, we see that $T$ is a totally ramified division algebra over $F^{\prime}$ of index $m / p_{1}^{\beta_{1}}$ by [JW, Corollary 2.6], and $T$ has exponent $n / p_{1}^{\alpha_{1}}$ by [TW, Theorem 4.7(i)]. Therefore $\bar{T}=\overline{F^{\prime}}=F$. Let $D=I \otimes_{F} T$. By Lemma 7,

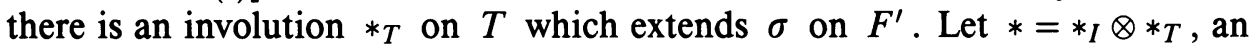
involution on $D$ extending $\sigma$ on $F^{\prime}$.

Theorem 10. The algebra $D$ defined above is a noncrossed product division algebra of index $m$ and exponent $n$, and $D$ contains a Baer ordering with respect to the involution *.

Proof. Since $I$ and $T$ have relatively prime indices, $D$ is a division algebra, and so $\operatorname{ind}(D)=m$. Also, $\exp (D)=\exp (I) \exp (T)=n$. Let $I^{\prime}=I \otimes_{F} F^{\prime}$. Then $I^{\prime}$ is inertial over $F^{\prime}$ and $D=I^{\prime} \otimes_{F^{\prime}} T$. The Henselian valuation on $F^{\prime}$ extends to $D$, and from the fundamental inequality we see that $\bar{D}=\overline{I^{\prime}}=I$ and $\Gamma_{D}=\Gamma_{T}$. Also, the involution on $\bar{D}$ induced by $*$ is $*_{I}$. If $v$ is the valuation on $F^{\prime}$, then $v \circ \sigma=v$, since $\left.v\right|_{K^{\prime}}$ extends uniquely to $F^{\prime}$ (as $K^{\prime}$ is Henselian). Thus by [W, Theorem], $v \circ *=v$ on $D$. Therefore $v$ is a *-valuation on $D$. We claim that $v$ is a smooth valuation. To see this, if $\gamma \in \Gamma_{D}=\Gamma_{T}$, then $\gamma=v(d)$ for some $d \in T$ with $d^{*}= \pm d$ (see $\S 2$ ). Moreover we may assume $d$ is symmetric by replacing $d$ by $i d$ if necessary. Since the residue automorphism $c_{d}$ is the identity for $d \in T$, we see that $d$ is a smooth element, hence $v$ is smooth. Therefore the Baer ordering on $I=\bar{D}$ can be lifted to $D$ by Theorem 1. (Alternatively, one could consider the (totally ramified) valuation on $D$ which is the composite of $v$ with the valuation on $I$ for which $I$ is totally ramified, and apply Corollary 2 to show that $D$ is Baer ordered.)

To finish the proof we need to show that $D$ is a noncrossed product. If not, then there is a maximal subfield $M$ of $D$ which is Galois over $F^{\prime}$. Let $G=\operatorname{Gal}\left(M / F^{\prime}\right)$. Let $N$ be a $p_{1}$-Sylow subgroup of $G$, and let $L$ be the fixed field of $N$. Then $\left[L: F^{\prime}\right]=m / p_{1}^{\beta_{1}}$ and $[M: L]=p_{1}^{\beta_{1}}$. If $I_{L}^{\prime}$ (resp. $T_{L}$ ) is the underlying division algebra of $I^{\prime} \otimes_{F^{\prime}} L$ (resp. $T \otimes_{F^{\prime}} L$ ), then $I_{L}^{\prime}$ and $T_{L}$ have relatively prime indices, $\operatorname{ind}\left(T_{L}\right)$ and $[M: L]$ are relatively prime, and $M$ splits $I_{L}^{\prime} \otimes_{L} T_{L}$. Therefore $T_{L}$ is split. Since $\left[L: F^{\prime}\right]=\operatorname{ind}(T)$, it follows 
that $L$ is a maximal subfield of $T$. Since $T$ is totally ramified over $F^{\prime}$, so is $L$; hence $L / F^{\prime}$ is Galois [TW, Proposition 1.4(iii)]. Thus $N$ is normal in $G$. Since $\operatorname{gcd}(|N|,|G / N|)=1$, the group $G$ is a semidirect product $N \rtimes H$ by the Schur-Zassenhaus theorem [R, p. 149]; in particular, $G$ contains a subgroup $H$ with $|G: H|=p_{1}^{\beta_{1}}$. If $E$ is the fixed field of $H$, then $\left[E: F^{\prime}\right]=p_{1}^{\beta_{1}}$; and so by a similar argument to that for $L$, we see that $E$ is a maximal subfield of $I^{\prime}$. Thus $E / F^{\prime}$ is unramified, so $[\bar{E}: F]=p_{1}^{\beta_{1}}$. Therefore $\bar{E}$ is a maximal subfield of $I=\overline{I^{\prime}}$. However, $\bar{E} \subseteq \bar{M} \subseteq \bar{D}=I$, so $\bar{M}$ is a maximal subfield of $I$. But $\bar{M} / F$ is Galois since $M / F^{\prime}$ is Galois and $\operatorname{char}(F)=0$, by [E, 14.5]. This means $I$ is a crossed product, which is false. Therefore $D$ is a noncrossed product.

We finish this paper with examples of indecomposable division algebras possessing a Baer ordering. We follow the method described in [JW, Remark 5.6]. Let $p$ be a prime, $m \geq 3$, and let $n$ be an integer with $m \geq n \geq \frac{m}{2}+1$. Construct the field $F$ exactly as in the beginning of this section, except with four $x_{j}$ and four $y_{j}$, and such that $\sigma\left(x_{j}\right)=x_{j}$ for $j=3,4$. Let $\omega_{j}$ be a primitive $p_{j}$ th root of unity in $F$. Let $t$ be the smallest integer greater than or equal to $m / 2$, and set

$$
\begin{gathered}
A_{1}=A_{\omega_{n}}\left(x_{1}+x_{2}, x_{1} x_{2} ; F\right) \otimes_{F} A_{\omega_{m-n}}\left(x_{3}, x_{4} ; F\right), \\
A_{2}=A_{\omega_{t}}\left(y_{1}, y_{2} ; F\right) \otimes_{F} A_{\omega_{m-t}}\left(y_{3}, y_{4} ; F\right) .
\end{gathered}
$$

Let $D$ be the underlying division algebra of $A_{1} \otimes_{F} A_{2}$. We equip $D$ with an involution $*$ extending $\sigma$ by Lemma 7 .

Proposition 11. The algebra $D$ above is an indecomposable division algebra of index $p^{m}$ and exponent $p^{n}$ which possesses a Baer ordering with respect to *.

Proof. That $D$ has index $p^{m}$ and exponent $p^{n}$, and is indecomposable, follows essentially from the argument in [JW, Remark 5.6]. (The argument given there for $m=4, n=3$ carries over here as the integers $n, m-n, t, m-t$ are all distinct and $D \otimes_{F} L_{1}=A_{\omega_{n}}\left(x_{1}, x_{2} ; L_{1}\right) \otimes_{L_{1}} A_{\omega_{m-n}}\left(x_{3}, x_{4} ; L_{1}\right)$, and $D \otimes_{F} L_{2}=A_{\omega_{t}}\left(y_{1}, y_{2} ; L_{2}\right) \otimes_{L_{2}} A_{\omega_{m-t}}\left(y_{3}, y_{4} ; L_{2}\right)$.) As in the case in Lemma 8, we see that $D$ has a *-valuation extending $v_{2}$ on $F$, such that $D / F$ is totally ramified. Therefore the Baer ordering on $\bar{D}=\bar{F}$ relative to $\bar{\sigma}$ lifts to a Baer ordering on $D$ relative to $*$.

Remark 1. The division algebra of Proposition 11 can be shown to be a crossed product. As noted in [JW, Remark 5.6], their construction of indecomposable division algebras can be modified to produce noncrossed products which are indecomposable, and similarly the construction above can be modified to produce indecomposable noncrossed products with a Baer ordering.

In all of the examples above, the involution involved is of the second kind. If $D$ has an involution of the first kind, then $\exp (D)=2$, and it is unknown whether there exist noncrossed products of exponent 2. However, in terms of indecomposability, Chacron and Wadsworth constructed in [CW] an indecomposable division algebra $D$ of index 8 and exponent 2 which has a $c$-valuation with respect to an involution $*$ of the first kind on $D$. They used the method of [JW] to construct $D$, and as in the examples of [JW] and ours, $D$ has a totally ramified valuation over $F=Z(D)$, and $\bar{F}$ is a formally real field. Thus 
any ordering on $\bar{F}=\bar{D}$ can be lifted to a Baer ordering of $D$ with respect to * by Theorem 1. Therefore there exist indecomposable Baer-ordered division algebras with involution of the first kind.

Remark 2. Since this paper was submitted, the authors ([MS]) have constructed indecomposable Baer ordered division algebras of index $p^{n}$ and exponent $p^{m}$ for all primes $p$ and integers $n>m \geq 1$ (except for $p=n=2$ and $m=1$, for which there are no indecomposable division algebras). The method used in [MS] is similar to that used in this paper, except that an indecomposable division algebra of the right index and exponent is combined with a totally ramified division algebra to obtain the desired examples.

\section{REFERENCES}

[B] R. Baer, Linear algebra and projective geometry, Academic Press, New York, 1952.

[ $\left.\mathrm{C}_{1}\right] \mathrm{T}$. Craven, Orderings and valuations on *-fields, Proc. of the Corvallis Conf. on Quadratic Forms and Real Algebraic Geometry, Rocky Mountain J. Math 19 (1989), 629-646.

$\left[\mathrm{C}_{2}\right]-$ Approximation properties for orderings on *-fields, Trans. Amer. Math. Soc. 310 (1988), 837-850.

[C $\left.\mathrm{C}_{3}\right]$, Places on *-fields and the real holomorphy ring, Comm. Algebra 18 (1990), 27912820.

[CW] M. Chacron and A. Wadsworth, On decomposing $c$-valued division rings, J. Algebra 134 (1990), 182-208.

[D] P. Draxl, Skew fields, London Math. Soc. Lecture Note Ser., vol. 81, Cambridge Univ. Press, Cambridge, 1983.

[E] O. Endler, Valuation theory, Springer-Verlag, New York, 1972.

$\left[\mathrm{H}_{1}\right]$ S. Holland, Orderings and square roots in *-fields, J. Algebra 46 (1977), 67-90.

$\left[\mathrm{H}_{2}\right]-$ - *-valuations and ordered *-fields, Trans. Amer. Math. Soc. 262 (1980), 219-243.

$\left[\mathrm{H}_{3}\right] \_$, Baer ordered *-fields of the first kind, Israel J. Math 57 (1987), 365-374.

[JW] B. Jacob and A. Wadsworth, A new construction of noncrossed product algebras, Trans. Amer. Math. Soc. 293 (1986), 693-721.

[L] T.-Y. Lam, Orderings, valuations and quadratic forms, CBMS Notes, No. 52, Amer. Math. Soc., Providence, RI, 1983.

[Le $\left.{ }_{1}\right]$ K. H. Leung, Baer orderings and weak *-orderings on *-fields, preprint.

$\left[\mathrm{Le}_{2}\right] \underset{1-22}{-}$, Strong approximation property for Baer orderings on *-fields, J. Algebra 165 (1994),

[MW] P. Morandi and A. Wadsworth, Baer orderings with noninvariant valuation ring, Israel $\mathbf{J}$. Math 68 (1989), 241-255.

[MS] P. Morandi and B. A. Sethuraman, Indecomposable division algebras with a Baer ordering, Comm. Algebra 22 (1994), 5401-5418.

[R] J. Rotman, The theory of groups: An introduction, 2nd ed., Allyn and Bacon, Boston, MA, 1973.

[TW] J.-P. Tignol and A. Wadsworth, Totally ramified valuations on firite-dimensional division algebras, Trans. Amer. Math. Soc. 302 (1987), 223-249.

[W] A. Wadsworth, Extending valuations to finite dimensional division algebras, Proc. Amer. Math. Soc. 98 (1986), 20-22.

Department of Mathematical Sciences, New Mexico State University, Las Cruces, NEW MEXICO 88003

E-mail address: pmorandi@nmsu.edu

Department of Mathematics, California State University-Northridge, Northridge, CALIFORNIA 91330

E-mail address: asethuraman@vax.csun.edu 